\title{
Reducing bias in population and landscape genetic inferences: The effects of sampling related individuals and multiple life stages
}

William Peterman, Emily R Brocato, Raymond D Semlitsch, Lori S Eggert

In population or landscape genetics studies, an unbiased sampling scheme is essential for generating accurate results, but logistics may lead to deviations from the sample design. Such deviations may come in the form of sampling multiple life stages. Presently, it is largely unknown what effect sampling different life stages can have on population or landscape genetic inference, or how mixing life stages can affect the parameters being measured. Additionally, the removal of siblings from a data set is considered best-practice, but direct comparisons of inferences made with and without siblings are limited. In this study, we sampled embryos, larvae, and adult Ambystoma maculatum from five ponds in Missouri, and analyzed them at 15 microsatellite loci. We calculated allelic richness, heterozygosity and effective population sizes for each life stage at each pond and tested for genetic differentiation $\left(F_{\mathrm{ST}}\right.$ and $D_{\mathrm{C}}$ ) and isolation-by-distance (IBD) among ponds. We tested for differences in each of these measures between life stages, and in a pooled population of all life stages. All calculations were done with and without sibling pairs to assess the effect of sibling removal. We also assessed the effect of reducing the number of microsatellites used to make inference. No statistically significant differences were found among ponds or life stages for any of the population genetic measures, but patterns of IBD differed among life stages. There was significant IBD when using adult samples, but tests using embryos, larvae, or a combination of the three life stages were not significant. We found that increasing the ratio of larval or embryo samples in the analysis of genetic distance weakened the IBD relationship, and when using $D_{C}$, the IBD was no longer significant when larvae and embryos exceeded $60 \%$ of the population sample. Further, power to detect an IBD relationship was reduced when fewer microsatellites were used in the analysis. 
1 Reducing bias in population and landscape genetic inferences: The effects of sampling related

2 individuals and multiple life stages

3

4 Running Head: Sampling multiple life stages

5

6

7 William E Peterman ${ }^{1,2 *}$, Emily R Brocato ${ }^{1}$, Raymond D Semlitsch ${ }^{1}$, Lori S Eggert ${ }^{1}$

8

9 ' ${ }^{1}$ University of Missouri, Division of Biological Sciences, Columbia, MO 65211

102 Present address: School of Environment and Natural Resources, The Ohio State University,

11 Columbus, $\mathrm{OH} 43210$

12

13

14 Address correspondence to:

15 William E. Peterman

16 School of Environment and Natural Resources, The Ohio State University, 2021 Coffey Road,

17210 Kottman Hall, Columbus, OH 43210-1085

18

19

20 


\section{Abstract}

22 In population or landscape genetics studies, an unbiased sampling scheme is essential for

23 generating accurate results, but logistics may lead to deviations from the sample design. Such

24 deviations may come in the form of sampling multiple life stages. Presently, it is largely

25 unknown what effect sampling different life stages can have on population or landscape genetic

26 inference, or how mixing life stages can affect the parameters being measured. Additionally, the

27 removal of siblings from a data set is considered best-practice, but direct comparisons of

28 inferences made with and without siblings are limited. In this study, we sampled embryos,

29 larvae, and adult Ambystoma maculatum from five ponds in Missouri, and analyzed them at 15

30 microsatellite loci. We calculated allelic richness, heterozygosity and effective population sizes

31 for each life stage at each pond and tested for genetic differentiation $\left(F_{\mathrm{ST}}\right.$ and $\left.D_{\mathrm{C}}\right)$ and isolation-

32 by-distance (IBD) among ponds. We tested for differences in each of these measures between

33 life stages, and in a pooled population of all life stages. All calculations were done with and

34 without sibling pairs to assess the effect of sibling removal. We also assessed the effect of

35 reducing the number of microsatellites used to make inference. No statistically significant

36 differences were found among ponds or life stages for any of the population genetic measures,

37 but patterns of IBD differed among life stages. There was significant IBD when using adult

38 samples, but tests using embryos, larvae, or a combination of the three life stages were not

39 significant. We found that increasing the ratio of larval or embryo samples in the analysis of

40 genetic distance weakened the IBD relationship, and when using $D_{\mathrm{C}}$, the IBD was no longer

41 significant when larvae and embryos exceeded $60 \%$ of the population sample. Further, power to

42 detect an IBD relationship was reduced when fewer microsatellites were used in the analysis. 


\section{Introduction}

45 An overarching goal of any study is to obtain accurate, unbiased estimates of the parameters of

46

47

48

49

50

51

52

53

54 55 interest. In population and landscape genetics, it is often recommended that 25-30 individuals be sampled from each population (Hale et al. 2012). For many species or systems, it is often easiest to meet these requirements by sampling early life stages (e.g., eggs or larvae) that can be found in abundance within a discrete area. However, many organisms experience extremely high mortality in these early life stages. Most amphibian, fish and insect species are characterized by Type III survivorship, in which a majority of young individuals will die before reaching sexual maturity, and the genetic characteristics of these life stages may differ from the few surviving adults due to the decrease in population size (Frankham 1996). While it may often be assumed that selection pressures that reduce population size act uniformly and randomly, selection may differentially affect individuals. For example, numerous studies have assessed the role of inbreeding and heterozygosity on individual fitness (e.g., Balloux et al. 2004; Ficetola et al. 2011; Harrison et al. 2011; Slate et al. 2004). Both of these population genetic attributes are particularly relevant in species of conservation concern, which often exist in small or isolated populations. Given the interaction between selection pressures and genetic diversity, it is not unreasonable to believe that population genetic measures may differ depending on the age or life stage of the sampled cohort.

2 Despite the potential problems with sampling different life stages, it is not uncommon for population or landscape genetic studies to combine samples from different cohorts or life stages, either because of convenience or necessity. Early life stages are often sampled because they are accessible, abundant, and cost-effective (Heyer et al. 1994). In amphibians, the extreme decline in individuals from early life stages to adults has been well-documented. Peterson et al. (1991), 
67 found a pre-metamorphic mortality rate of 99\% in ringed salamanders (Ambystoma annulatum),

68 Shoop (1974) found that pre-metamorphic mortality rates ranged from $87-99 \%$ in spotted

69 salamanders (A. maculatum), and Berven (1990) recorded pre-metamorphic mortality rates

70 ranging from $97-99 \%$ in wood frogs (Rana sylvatica). The drastic decline in abundance can also

71 be seen in fish and insects. Dahlberg (1979) found a mortality rate of $>99 \%$ in the eggs of many

72 fish species, while a study of the southern green stink bug (Nezara viridula) found mortality rates

73 to be as high as 96\% (Kiritani \& Nakasuji 1967). As such, when early life stages are sampled to

74 make inferences about the adult population, biased conclusions may result (Allendorf \& Phelps

75 1981; Goldberg \& Waits 2010). Obtaining unbiased estimates of genetic diversity is particularly

76 critical for management and conservation of species.

77 Sampling animals from the field is often opportunistic due to the availability of the target

78 species. Environmental factors, stochastic events, or the timing of offspring can alter when a life

79 stage becomes available, if it can be found at all (Mullins et al. 2004). In these cases, researchers

80 often need to stray away from their sampling scheme and target life stage, and collect other life

81 stages to reconcile the sample size gap (e.g., Lee-Yaw et al. 2009; Richardson 2012). Despite the

82 relative commonness of these sampling realities, the effect of mixing life stages in population

83 and landscape genetic analyses has not been explicitly addressed. The sampling of full siblings

84 has been shown to affect the estimates of population genetic parameters (Goldberg \& Waits

85 2010). When sampling amphibians, field researchers have the highest probability of collecting

86 sibling pairs within larvae (Goldberg \& Waits 2010); related larvae are often spatially clustered,

87 and samples collected at a specific location may be biased towards a single family group

88 (Hansen et al. 1997). If researchers are unaware that family groups are being sampled, the

89 genetic structure of the family could be misinterpreted as population structure within the 
90 panmictic population (Anderson \& Dunham 2008). To prevent misinterpretations and avoid

91 biased population genetic parameter estimates, it has been suggested that samples be screened

92 prior to analysis, and full siblings removed (Goldberg \& Waits 2010).

93 To date, only Goldberg \& Waits (2010) have empirically tested the effects of sampling

94 different life stages in amphibians (one frog and one salamander species) and quantified the

95 importance of removing full siblings prior to analysis. The primary objective of this study was to

96 determine the effects of pooling different life stages on population and landscape genetic

97 inferences. Additionally, we sought to extend the findings of Goldberg \& Waits (2010) to

98 determine the effect of sampling three life stages in amphibians: adults, embryos, and larvae, on

99 population and landscape genetic inferences. We assessed these objectives both with and without

100 full-sibling pairs present in the data set, and also assessed how inferences are affected by

101 reducing the number of microsatellites used for analysis. We predicted that the random mixing of

102 life stages would result in genetic parameter estimates that did not differ from estimates of

103 individual life stages. However, we predicted that there would be significant biases present when

104 sampling different life stages as certain alleles are likely to be over-represented in the embryonic

105 and larval life stages. Finally, we predicted that the removal of siblings from the data set would

106 significantly alter population and landscape genetic estimates by increasing average allelic

107 richness and heterozygosity within a sampled population, as well as increasing the average

108 genetic differentiation among populations.

109

110 Materials and methods

111 Ethics Statement - This research was conducted in compliance with all laws and regulations for

112 the state of Missouri and the USA, and was conducted under Missouri Wildlife Collector's 
113 permit 15584. Sampling methods were approved by the University of Missouri Animal Care and

114 Use Committee (Protocol 7403).

115

116 Data availability - All data and code used in this study can be accessed from Figshare at 117 https://dx.doi.org/10.6084/m9.figshare.1621318.v2.

118

119 Literature review - To determine how researchers are currently collecting tissue samples from

120 amphibians with complex life cycles, we conducted a literature search of the Scopus database of

121 population and landscape genetic studies of amphibians. We used the search terms "amphibia*"

122 (occurring in the title, abstract, or keywords), "microsatellite*" (occurring in all fields), and NOT

123 "reptil*" (occurring in the title, abstract, keywords) and limited the search to findings from

124 Molecular Ecology, Conservation Genetics, Heredity, Biological Journal of the Linnean Society,

125 Amphibia-Reptilia, Animal Conservation, Molecular Ecology Resources, Evolution, Plos One,

126 or Journal of Zoology published through December 2012. For each study, we determined if

127 different life stages were sampled and if the study gave an indication as to whether sampling

128 multiple life stages influenced analysis or inferences made from the data.

129

130 Sampling-Our study was conducted at Daniel Boone Conservation Area (DBCA), in Warren

131 County, Missouri, U.S.A. (Fig. 1). This 1,424 ha area is situated on the upper Ozark Plateau

132 physiographic region and is characterized by mature (80-100 years old) second-growth forest

133 with an overstory dominated by oak (Quercus spp.) and hickory (Carya spp.), with varying

134 amounts of sugar maple (Acer saccharum) and red cedar (Juniperus virginiana) in the understory

135 (Semlitsch et al. 2009). There are $>40$ fishless manmade ponds that are, on average, separated by 
$1362,000 \mathrm{~m}(246-3,900 \mathrm{~m})$ (Peterman et al. 2013b). Only man-made ponds are known to still exist

137 on the DBCA landscape. We sampled adults, embryos, and larvae of Ambystoma maculatum

138 (spotted salamander) from five ponds at DBCA (Fig. 1). Each of these ponds have been the focus

139 of previous amphibian research at DBCA (e.g., Hocking et al. 2008; Semlitsch et al. 2014), and

140 have similar surface area $\left(160-330 \mathrm{~m}^{2}\right)$, depth $(<1.2 \mathrm{~m})$, age $(27-47 \mathrm{yrs})$, and permanent

141 hydroperiod. We sought to collect 25 adult and embryo samples and 30 larval samples from each

142 pond. Adult salamanders were captured in mesh funnel traps placed in breeding ponds in March

1432013 , and tissue samples were obtained by removing $0.5 \mathrm{~cm}$ of tail tissue. Following oviposition,

144 we sampled embryos by collecting a single embryo per clutch in April 2013. In June 2013 larvae

145 were captured with dip nets, and to minimize the sampling of siblings, we collected larvae from

146 the entire perimeter of each pond. Upon collection in the field, each tissue sample was placed in

$14795 \%$ ethanol and stored at $-20^{\circ} \mathrm{C}$ until DNA extraction.

148

149 Lab techniques - DNA was extracted from tissue using chelex-based resin (InstaGene, BioRad).

150 Approximately $2.5 \mathrm{~mm} \times 2.5 \mathrm{~mm}$ of tissue was finely chopped with a sterile razor and was

151 incubated at $60^{\circ} \mathrm{C}$ for $2 \mathrm{hrs}$ in $250 \mu \mathrm{L}$ of InstaGene, vortexed, incubated for $20 \mathrm{~min}$ at $100^{\circ} \mathrm{C}$,

152 then vortexed again. Following centrifugation, a $100 \mu \mathrm{L}$ aliquot was removed and used as

153 template DNA and the remainder was kept at $-20^{\circ} \mathrm{C}$ (Peterman et al. 2012). Nineteen tetra-

154 nucleotide microsatellite loci were amplified using PCR; primers were fluorescently 5' labeled

155 with FAM, NED, VIC, and PET and arranged into two multiplex reactions (Peterman et al.

156 2013a). Negative controls were included in all reactions to detect contamination of reagents.

157 Amplification products were sized on an ABI 3730xl DNA Analyzer (Applied Biosystems,

158 Foster City, CA, USA) using Liz 600 size standard at the University of Missouri DNA Core 
159 Facility, and results were scored using GENEMARKER (v.1.97; Softgenetics, State College, PA, 160 USA).

161

162 Differences among life stages - Before proceeding with analyses we created a data set free of 163 full sibling pairs using COLONY (Wang 2012). For our COLONY analyses, both male and

164 female mating were set to polygamous without inbreeding. We conducted a long run with full 165 likelihood and high precision and did not include a sibship prior. We excluded siblings from the 166 analysis such that all sites only had one individual per family group. Values for $F_{\mathrm{ST}}$ and allelic 167 richness (rarefied to our smallest sample) were calculated with the R package hierfstat (Goudet 168 2013), observed heterozygosity and chord distance $\left(D_{\mathrm{C}}\right)$ were calculated with the R package 169 adegenet (Jombart \& Ahmed 2011), and effective population size estimates $\left(\mathrm{N}_{\mathrm{e}}\right)$ were made 170 using the linkage disequilibrium method implemented in COLONY (Wang 2012). The

171 proportion of siblings removed from each life stage at each pond was also calculated. To

172 determine the effect of sibling removal, we also calculated summary statistics $\left(\mathrm{H}_{\mathrm{o}}, \mathrm{A}_{\mathrm{r}}, F_{\mathrm{ST}}, D_{\mathrm{C}}\right)$

173 for each life stage with siblings present. All population genetic measures were compared among

174 life stages and between estimates made with and without siblings using analysis of variance 175 (ANOVA) and paired t-tests. Due to small sample sizes, we bootstrapped our ANOVA analyses 176 and conducted permutation t-tests to more robustly assess differences among life stages and 177 removal of siblings.

179 Effect of mixing life stages - Prior to pooling life stages together, we conducted a second 180 removal of related individuals using the COLONY settings described above (Wang 2012).

181 Specifically, we found and removed parent-offspring and embryo-larvae sibling pairs within 
182 each pond. All unrelated individuals of all life stages were pooled by pond of origin to make five

183 mixed-tissue populations. From these populations, we randomly sampled 25 individuals using

184 the R package hierfstat (Goudet 2013) in R (R Core Team 2013). This bootstrap resampling

185 procedure was repeated 1,000 times (both with and without siblings), and the mean and 95\%

186 confidence intervals were calculated for $\mathrm{H}_{\mathrm{o}}, \mathrm{A}_{\mathrm{r}}, F_{\mathrm{ST}}$, and $D_{\mathrm{C}}$.

187

188 Isolation-by-distance analysis - For the isolation-by-distance (IBD) analysis we conducted

189 simple Mantel tests correlating the genetic distance $\left(F_{\mathrm{ST}}\right)$ with the Euclidean distance between

190 ponds. This test was repeated for all life stages, with and without siblings, and significance was

191 assessed using 100,000 permutations using the R package ecodist (Goslee \& Urban 2007). We

192 tested for IBD in the mixed sample population, and calculated the mean and 95\% confidence

193 interval for both the Mantel $r$ correlation statistic and the associated P-value based on the 1,000

194 bootstrap iterations. Because we found a significant IBD relationship when using adult-only

195 tissue samples (see Results), we further assessed how the IBD relationship changed with the

196 inclusion of larval and embryo samples. For this analysis, we varied the proportion of larval and

197 embryo samples included with our adult samples. This was assessed at proportions ranging from

1980 (no larval or embryo samples) to 1 (only larval and embryo samples) at increments of 0.05 . At

199 each increment, we assessed the mean and 95\% confidence intervals of the Mantel $r$ and the

200 corresponding P-value based on 1,000 bootstrap samples of the data. We used the data set

201 without siblings for this analysis and sampled each population to the minimum adult sample size $202(n=18)$.

203 
204 Number of microsatellite loci - Concurrent with assessing the effects of mixing life stages, we 205 also assessed the effects of reducing the number of microsatellites used in an analysis. Within the 206 bootstrapping procedure for assessing the proportion of larval and embryo samples described 207 above, we sub-sampled our microsatellite data set to include either 5, 10, or all 15 of the 208 microsatellites. At each bootstrap iteration at each mixture proportion, microsatellites were 209 randomly chosen to calculate Mantel $r$ and the corresponding P-value. We also calculated the 210 observed heterozygosity and allelic richness at each mixture proportion and for each level of 211 microsatellite subsampling. These estimates were averaged over all populations sampled.

\section{Results}

214 Literature review - We found that 20 out of 95 (21\%) of studies meeting our search criteria on 215 Scopus conducted population or landscape genetic analyses of amphibian species using mixed 216 tissue sampling (searched on 13 February 2016). Five of these studies stated that one life stage 217 was sampled only when the target life stage was not available (Beebee \& Rowe 2000; Lee-Yaw 218 et al. 2009; Lee-Yaw et al. 2008; Munwes et al. 2010; Richardson 2012). None of these studies

219 made attempts to check or correct for the effects of mixing life stages in their analyses, although

220 it was common for siblings to be removed prior to analysis.

221

222 Sample summary - We collected 24-25 adults and 19-27 embryos from each of the five ponds, 223 and 29-36 larvae from three of the five ponds (Supplement 1). We were unable to sample larvae

224 from two of the ponds due to high embryo mortality. Of the original 19 screened primers, two 225 loci $\left(A m_{-} 13, A m_{-} 60\right)$ were not polymorphic, and two loci $\left(A m_{-} 33, A m_{-} 43\right)$ showed very little 226 polymorphism and deviated significantly from expected heterozygosity values under Hardy 
227 Weinberg equilibrium (HWE). These four loci were removed from the dataset and all population

228 genetic statistics were calculated using the remaining 15 loci (Supplement 2). No other loci or

229 populations deviated from Hardy-Weinberg equilibrium or were significantly linked. Overall, we

$230 \mathrm{had}<0.5 \%$ missing data.

231

232 Statistical summary - For all tests, none of the population genetic parameters differed

233 significantly among life stages (bootstrap ANOVA P-value $>0.05$ ) or within ponds and among

234 life stages (permutation t-test P-value $\geq 0.25$; Fig. 2, Tables $1-2$ ), regardless of whether or not

235 siblings were present in the data. The proportion of samples removed due to sibship was nearly

236 significant (bootstrap P-value $=0.053$, Fig. 2f), with a greater proportion of field-collected

237 samples being omitted from larvae. There was also an increase in the estimated mean $F_{\text {ST }}$

238 calculated in the larval and embryo data as compared to adults (Fig. 2c), but we note that this

239 increase was not significant. However, this trend was not observed when genetic distance was

240 measured using allele frequencies $\left(D_{\mathrm{C}}\right.$, Fig. $\left.2 \mathrm{~d}\right)$. Values of population genetic summary statistics

241 calculated on data sets with siblings removed are given as the mean ( \pm standard deviation).

242 Effective population size among ponds averaged $87.4( \pm 25.28)$ for adults, $82.4( \pm 25.58)$ for

243 embryos, and $64( \pm 4.32)$ for larvae. Average rarefied allelic richness of adults was $3.83( \pm 0.22)$,

$2443.95( \pm 0.23)$ for embryos, and $4.13( \pm 0.20)$ for larvae. The average observed heterozygosity was

$2450.53( \pm 0.01)$ for adults, $0.51( \pm 0.03)$ for embryos, and $0.51( \pm 0.01)$ for larvae. On average, we

246 removed $33.2 \%( \pm 0.09)$ of larval samples due to sibship, while only $13 \%( \pm 0.084)$ of adult and

$24714.98 \%( \pm 0.09)$ of embryo samples were removed. With siblings removed, pairwise genetic

248 distances between ponds measured using $F_{\mathrm{ST}}$ averaged $0.011( \pm 0.008)$ in adults, $0.021( \pm 0.007)$ in 
249 larvae, and $0.019( \pm 0.014)$ in embryos, while $D_{C}$ averaged $0.226( \pm 0.025)$ in adults, 0.237

$250( \pm 0.011)$ in larvae, and $0.240( \pm 0.030)$ in embryos (Table 3$)$.

251 When comparing each life stage at each summary metric, we found no significant

252 differences between data containing the sibling pairs and data with removed sibling pairs

253 (permutation t-test $\mathrm{P}$-value $\geq 0.19$; Fig. 2, Tables $1-2$ ). We found that the mixing of life stages

254 resulted in genetic estimates of $\mathrm{A}_{\mathrm{r}}, \mathrm{H}_{\mathrm{o}}, F_{\mathrm{ST}}$, and $D_{\mathrm{C}}$ that, on average, did not differ from estimates 255 made for each specific life stage (Tables 1-2). There were, however, up to three pond-pair $F_{\text {ST }}$ 256 values that fell outside of the bootstrapped 95\% confidence intervals (Table 3 ). When genetic 257 distance was measured using $D_{\mathrm{C}}$, only one pond-pair fell outside of the bootstrapped confidence 258 interval. In general, there was a greater frequency of pairwise genetic distance measures based on 259 embryo samples to fall outside of the mixed sample confidence interval. For both $F_{\mathrm{ST}}$ and $D_{\mathrm{C}}$, the 260 removal of siblings resulted in more pairwise estimates falling outside of the mixed sample

261 confidence interval (Table 3). Due to sample size, clear inferences cannot be drawn from larvae.

262 With regard to IBD, only tests using adult samples (with and without siblings) resulted in 263 significant relationships (Table 4), and the IBD relationship was stronger when genetic distance 264 was measured using $D_{\mathrm{C}}$ (Fig. 3). IBD tests from embryos or larvae had much lower Mantel $r$ 265 correlations and were not significant. The mixing of tissue samples resulted in non-significant 266 IBD tests when using $F_{\mathrm{ST}}\left(p>0.05\right.$; Table 4), but had little effect when using $D_{\mathrm{C}}$ (Table 4). Our assessment of increasing the proportion of larval or embryo tissue samples clearly

268 demonstrated that the calculated Mantel $r$ decreases as the proportion of larval and embryo 269 samples increases (Fig. 3). This pattern was consistent regardless of whether genetic distance 270 was measured using $F_{\mathrm{ST}}$ or $D_{\mathrm{C}}$. Correspondingly, the average P-value of the Mantel test

271 increased as the proportion of larval and embryo samples increased. Further, reducing the 
272 number of microsatellites resulted in a reduced Mantel correlation and increased P-value (Fig. 3).

273 When using all 15 microsatellites for this analysis, the mean bootstrapped P-value for tests using

$274 F_{\text {ST }}$ started at $0.057(0.055-0.60)$ for adult-only samples, and increased to $0.231(0.223-0.239)$

275 for larvae/embryo-only samples (Fig. 3b). In contrast, the P-value for Mantel tests with $D_{\mathrm{C}}$

276 started at $0.019(0.018-0.020)$ when only adults were included and increased to $0.146(0.140-$

277 0.152) without adult samples (Fig. 3d). The 0.05 P-value threshold is passed when the proportion

278 of larvae and embryos in the sample reaches $0.60-0.65$ (P-values $=0.047-0.054$, respectively).

279 When 10 microsatellites are used, the 0.05 threshold is exceeded when the proportion of larvae 280 and embryos reaches $0.30-0.35$ (P-values $=0.047-0.052$, respectively). The IBD relationship

281 was not significant at any mixture proportion when only five microsatellites were used.

282 There was a trend for the mean heterozygosity to decrease and mean allelic richness to 283 increase as the proportion of larvae and embryos increased, but we note that these trends occur 284 over a very limited parameter space (Fig. 4). Adult-only samples had a mean observed 285 heterozygosity of 0.533 and mean allelic richness of 3.89, while larvae/embryo-only samples had 286 a mean observed heterozygosity of 0.521 and mean allelic richness of 4.11 . The number of 287 microsatellites used in the analysis did not affect the mean estimate of heterozygosity or allelic 288 richness, although precision in the bootstrap estimates was greater with more microsatellites.

290 Discussion

291 Our literature search revealed that mixed tissues have been used in about one fifth of amphibian 292 population genetic studies using microsatellites, despite the lack of knowledge concerning the 293 effects that this may have on population or landscape genetic inferences. Collection of genetic 294 samples from the field is likely influenced by numerous factors, such as the timing of life stage 
295 development and accessibility to sampling sites. Many of the studies that mixed tissues did so

296 when the target life stage was not found and minimum sample requirements had to be met.

297 Decisions made during sampling can undoubtedly influence the inferences made, as genetic

298 variation within structured populations can vary spatially, temporally, or as a result of life history

299 (Anderson \& Dunham 2008; Schwartz \& McKelvey 2009; Scillitani et al. 1996).

300 In our study, however, we found little evidence for adverse effects of including siblings

301 or with mixing tissue samples collected from different life stages when assessing levels of allelic

302 richness, heterozygosity, or effective population size. Contrary to Goldberg \& Waits (2010), it

303 may not always be necessary to remove siblings from a dataset to achieve unbiased population

304 genetic estimates. Nonetheless, we urge researchers to proceed with caution when there is a high

305 likelihood that full-siblings are present in a data set, and to carefully assess the tradeoffs between

306 power and precision. We found that estimates of pairwise $F_{\mathrm{ST}}$, although not statistically different,

307 differed substantially between life stages as well as from the mixed-tissue sample. However, this

308 pattern was not observed when pairwise genetic distance was measured using $D_{\mathrm{C}}$. The greatest

309 impact of mixing life stages was evident in our tests of IBD among pond pairs. Only the tests

310 using adults were significant, while larvae-only, embryo-only, and mixed-tissue samples showed

311 little correlation with distance. The IBD relationship was strongest when genetic distance was

312 measured using $D_{\mathrm{C}}$, and we found that the IBD relationship seen in adult-only samples decreased

313 as the proportion of larvae and embryos included in the population sample increased. In our

314 dataset, the IBD relationship, when assessed with $D_{\mathrm{C}}$, remained significant until the frequency of

315 larvae and embryos in the population sample exceeded $60 \%$. However, our power to detect a

316 significant IBD relationship was substantially affected by the number of microsatellites used.

317 When 10 of the 15 microsatellites were used, the IBD relationship became non-significant when 
318 the frequency of larvae and embryos exceeded 30\%, and no significant IBD relationship was

319 evident when only five microsatellites were used. As such, our results suggest the greatest effects

320 of mixing different life stages may be evident in landscape genetic analyses assessing pairwise

321 distances among populations, with different conclusions potentially being drawn from adult-only

322 samples as compared to larvae, embryo, or mixed-tissue samples. Further, such relationships

323 may be sensitive to the genetic distance statistic used as well as the number or polymorphism of

324 the microsatellites used.

325 Contrary to our predictions, we did not observe significant biases in our population

326 genetic measures among life stages or with the exclusion of full siblings from the data. This is

327 counter to the findings of Goldberg \& Waits (2010) who found that skewed estimates between

328 larval and adult population genetic measures were eliminated or reduced when full siblings were

329 removed from the larval sample. As in the analysis of mixed-tissue samples, the greatest

330 differences were observed in relation to genetic differentiation measured by $F_{\mathrm{ST}}$, which increased

331 (insignificantly) after the removal of full siblings from the data, as well as from adults to

332 embryos, to larvae. These increases in genetic differentiation in the embryonic and larval stages,

333 as compared to the adults is predicted by population genetic theory (Allendorf \& Phelps 1981),

334 and has been empirically demonstrated in Columbia spotted frogs (Rana luteiventris) (Goldberg

$335 \&$ Waits 2010).

336 The clearest result from our study was the proportion of samples that had to be removed

337 from each life stage due to redundancy of siblings. It is actually quite unlikely that we sampled

338 full sibling adults given their life history and longevity (Petranka 1998), but we chose to remove

339 these putative siblings from our data set for consistency of methods among life stages. We note,

340 however, that Goldberg \& Waits (2010) did not test for or remove sibling pairs from their adult 
341 samples. In our study, up to $40 \%$ of larval samples were found to be from sibling pairs, which

342 would have to be removed if their inclusion biased genetic parameter estimates. Although larvae

343 are often the most readily accessible and conveniently sampled life stage, this represents an

344 inefficient use of resources. When possible, it may be prudent to avoid sampling larvae.

345 Ultimately, the choice of which life stage or stages to sample will be idiosyncratic to the study

346 and system. We have demonstrated that inferences differed minimally and insignificantly

347 between larvae, embryos, and adults, but we note that both larvae and adults can be sampled

348 non-destructively, which may become a factor in deciding which life stage to sample for some

349 species.

In our study, we assessed IBD through the use of simple Mantel tests. We readily

351

352

353

354

355

356

357

358

acknowledge the limitations and criticisms of the Mantel test for making robust inference (e.g.,

Guillot \& Rousset 2013; Legendre et al. 2015). However, we feel that for our limited data set and ultimate goal of assessing relative differences and patterns between life stages, mixed life stage samples, and genetic distance measures, the simple Mantel test was sufficient and provides an appropriate cautionary caveat for future researchers using any method. More rigorous methods such as distance-based redundancy analysis (Legendre \& Anderson 1999), multiple regression of distance matrices (Holzhauer et al. 2006), distance-based Moran’s eigenvector maps (Legendre \& Legendre 2012), or mixed effects models fit with an appropriate error structure (Clarke et al. 2002) should preferentially be used over Mantel tests in future studies seeking to estimate the effects of landscape features on genetic differentiation.

To our knowledge, our study is the first attempt to determine how the sampling and mixing of different life stages affects genetic parameter estimates. It is unclear how differences in life history (e.g., life span, breeding site fidelity, reproductive strategy, etc.) alter the effects of 
364 sampling different life stages, as we currently do not have a mechanistic explanation for the

365 patterns we observed. We found that mixed-tissue samples can lead to different conclusions

366 when conducting spatial analyses, such as IBD, and these results would likely extend to more

367 complex landscape genetic analyses as well. As such, we caution researchers to carefully

368 consider the implications of mixing samples collected from multiple life stages. Our finding that

369 population genetic parameters differed little with the removal of siblings or mixing of life stages

370 was surprising and contrary to our predictions. In our study, we had a relatively small sample

371 size from which to draw inference, although we note that it is not much smaller than that used by

372 Goldberg \& Waits (2010) who assessed eight populations of $R$. luteiventris and four populations

373 of $A$. macrodactylum. We do note that the power to infer differences is likely greater in our study

374 due to the large number of polymorphic microsatellites used, which was reinforced by our results

375 of subsampling the number of genetic markers used. Perhaps of greater importance is the fact

376 that our populations are relatively close together (maximum distance $=3,200 \mathrm{~m}$ ) and situated

377 within continuous forest habitat. In contrast, Goldberg \& Waits (2010) sampled populations

378 separated by $2.7-18.5 \mathrm{~km}$ of agricultural matrix. Further, the populations included in our study

379 are very robust, with recorded breeding aggregations of several hundred individuals (Semlitsch

380 unpublished data).

$381 \quad$ Like Goldberg \& Waits (2010), we suggest that future studies include a pilot phase to

382 assess the effects of sampling different life stages to meet the objectives of the specific project.

383 An important consideration for any population or landscape genetic study is the target

384 demographic group for which inferences are desired. If quantifying movement or connectivity of

385 populations is the main objective of a study, then sampling adult life stages may provide the

386 most accurate inferences. If the study objectives are to quantify the distribution of genetic 
387 diversity, then sampling of embryos appears to be the most efficient use of resources. However, 388 nearly equivalent estimates can be obtained from larvae, and as demonstrated in this study, the 389 presence of siblings may not result in biased estimates. Ultimately, the mechanisms underlying 390 the patterns observed in this study are not known, but this may be a fruitful avenue for future 391 research to explore through simulation modeling to better understand how variation life history 392 characteristics and sampling scenarios affect population and landscape genetic inference. 393

\section{Acknowledgements}

395 We thank T. Luhring for assistance in the field. 


\section{References}

397 Allendorf FW, and Phelps SR. 1981. Use of allelic frequencies to describe population structure. Canadian Journal of Fisheries and Aquatic Sciences 38:1507-1514.

399

400

401

402

403

404

405

406

407

408

409

410

411

412

413

414

415

416

417

418

Anderson EC, and Dunham KK. 2008. The influence of family groups on inferences made with the program Structure. Molecular Ecology Resources 8:1219-1229. DOI: 10.1111/j.1755-0998.2008.02355.x.

Balloux F, Amos W, and Coulson T. 2004. Does heterozygosity estimate inbreeding in real populations? Molecular Ecology 13:3021-3031.

Beebee TJC, and Rowe G. 2000. Microsatellite analysis of natterjack toad Bufo calamita Laurenti populations: Consequences of dispersal from a Pleistocene refugium. Biological Journal of the Linnean Society 69:367-381.

Berven KA. 1990. Factors affecting population fluctuations in larval and adult stages of the wood frog (Rana sylvatica). Ecology 71:1599-1608.

Clarke RT, Rothery P, and Raybould AF. 2002. Confidence limits for regression relationships between distance matrices: Estimating gene flow with distance. Journal of Agricultural, Biological, and Environmental Statistics 7:361-372. DOI: 10.1198/108571102320.

Dahlberg MD. 1979. A review of survival rates of fish eggs and larvae in relation to impact assessments. Marine Fisheries Review 41:1-12.

Ficetola GF, Garner TWJ, Wang J, and De Bernardi F. 2011. Rapid selection against inbreeding in a wild population of a rare frog. Evolutionary Applications 4:30-38. DOI: 10.1111/j.1752-4571.2010.00130.x.

Frankham R. 1996. Relationship of genetic variation to population size in wildlife. Conservation Biology 10:1500-1508. 
419 Goldberg CS, and Waits LP. 2010. Quantification and reduction of bias from sampling larvae to 420 infer population and landscape genetic structure. Molecular Ecology Resources 10:304421 313. DOI: $10.1111 /$ j.1755-0998.2009.02755.x.

422 Goslee SC, and Urban DL. 2007. The ecodist package for dissimilarity-based analysis of 423 ecological data. Journal of Statistical Software 22:1-19.

424 Goudet J. 2013. hierfstat: Estimation and tests of hierarchical F-statistics. R package version 425 0.04-10. http://CRAN.R-project.org/package=hierfstat.

426

427

428

429

430

431

432

433

434

435

436

437

438

439

440

441

Guillot G, and Rousset F. 2013. Dismantling the Mantel tests. Methods in Ecology and Evolution 4:336-344. DOI: 10.1111/2041-210x.12018.

Hale ML, Burg TM, and Steeves TE. 2012. Sampling for microsatellite-based population genetic studies: 25 to 30 individuals per population is enough to accurately estimate allele frequencies. PLoS ONE 7:e45170.

Hansen MM, Nielsen EE, and Mensberg KLD. 1997. The problem of sampling families rather than populations: relatedness among individuals in samples of juvenile brown trout Salmo trutta L. Molecular Ecology 6:469-474. DOI: 10.1046/j.1365-294X.1997.t01-100202.x.

Harrison XA, Bearhop S, Inger R, Colhoun K, Gudmundsson GA, Hodgson D, McElwaine G, and Tregenza TOM. 2011. Heterozygosity-fitness correlations in a migratory bird: an analysis of inbreeding and single-locus effects. Molecular Ecology 20:4786-4795. DOI: 10.1111/j.1365-294X.2011.05283.x.

Heyer WR, Donnelly MA, McDiarmid RW, and Hayek L-AC. 1994. Measuring and monitoring biological diversity: standard methods for amphibians. In: Foster MS, editor. Biological diversity handbook series. Washington, D.C.: Smithsonian Institution Press. p 364. 
442 Hocking DJ, Rittenhouse TAG, Rothermel BB, Johnson BR, Conner CA, Harper EB, and 443 Semlitsch RD. 2008. Breeding and recruitment phenology of amphibians in Missouri 444 oak-hickory forests. American Midland Naturalist 160:41-60.

445 Holzhauer SIJ, Ekschmitt K, Sander AC, Dauber J, and Wolters V. 2006. Effect of historic 446 landscape change on the genetic structure of the bush-cricket Metrioptera roeseli. $447 \quad$ Landscape Ecology 21:891-899.

448 Jombart T, and Ahmed I. 2011. adegenet 1.3-1: new tools for the analysis of genome-wide SNP 449 data. Bioinformatics 27:3070-3071. DOI: 10.1093/bioinformatics/btr521.

450 Kiritani K, and Nakasuji F. 1967. Estimation of the stage-specific survival rate in the insect 451 population with overlapping stages. Researches on Population Ecology 9:143-152.

452 Lee-Yaw JA, Davidson A, McRae BH, and Green DM. 2009. Do landscape processes predict 453 phylogeographic patterns in the wood frog? Molecular Ecology 18:1863-1874. DOI:

454 10.1111/j.1365-294X.2009.04152.x.

455

Lee-Yaw JA, Irwin JT, and Green DM. 2008. Postglacial range expansion from northern refugia 456 by the wood frog, Rana sylvatica. Molecular Ecology 17:867-884.

457

458

459

460

461

462

463
Legendre P, and Anderson MJ. 1999. Distance-based redundancy analysis: Testing multispecies responses in multifactorial ecological experiments. Ecological Monographs 69:1-24. DOI: 10.1890/0012-9615(1999)069[0001:DBRATM]2.0.CO;2.

Legendre P, Fortin M-J, and Borcard D. 2015. Should the Mantel test be used in spatial analysis? Methods in Ecology and Evolution 6:1239-1247. DOI: 10.1111/2041-210X.12425.

Legendre P, and Legendre L. 2012. Chapter 12 - Ecological data series. In: Pierre L, and Louis L, eds. Developments in Environmental Modelling: Elsevier, 711-783. 
464 Mullins ML, Pierce BA, and Gutzwiller KJ. 2004. Assessment of quantitative enclosure 465 sampling of larval amphibians. Journal of Herpetology 38:166-172. DOI:

466 $10.2307 / 1566210$.

467 468 469 470

471

472

473

474

475

476

477

478

479

480

481

482

483

484

485

486 change in genetic diversity down the core-edge gradient in the eastern spadefoot toad (Pelobates syriacus). Molecular Ecology 19:2675-2689.

Peterman WE, Brocato ER, Pauley LR, Stuart EC, Semlitsch RD, and Eggert LS. 2013a. Development and characterization of 18 microsatellite loci for the spotted salamander (Ambystoma maculatum) using paired-end Illumina shotgun sequencing. Conservation Genetics Resources 5:989-991. DOI: 10.1007/s12686-013-9950-4.

Peterman WE, Connette GM, Spatola BN, Eggert LS, and Semlitsch RD. 2012. Identification of polymorphic loci in Ambystoma annulatum and review of cross-species microsatellite use in the genus Ambystoma. Copeia 2012:570-577. DOI: 10.1643/ch-11-001.

Peterman WE, Rittenhouse TAG, Earl JE, and Semlitsch RD. 2013b. Demographic network and multi-season occupancy modeling of Rana sylvatica reveal spatial and temporal patterns of population connectivity and persistence. Landscape Ecology 28:1601-1613. DOI: 10.1007/s10980-013-9906-9.

Peterson CL, Wilkinson RF, Don M, and Holder T. 1991. Premetamorphic survival of Ambystoma annulatum. Herpetologica 47:96-100.

Petranka JW. 1998. Salamanders of the United States and Canada. Washington D. C.: Smithsonian Institution Press.

R Core Team. 2013. R: A language and environment for statistical computing. R Foundation for Statistical Computing, Vienna, Austria. http://www.R-project.org/. 
487 Richardson JL. 2012. Divergent landscape effects on population connectivity in two co-

488 occurring amphibian species. Molecular Ecology 21:4437-4451. DOI: 10.1111/j.1365-

489 294X.2012.05708.x.

490 Schwartz MK, and McKelvey KS. 2009. Why sampling scheme matters: The effect of sampling

491 scheme on landscape genetic results. Conservation Genetics 10:441-452.

492

493

494

495

496

497

498

499

500

501

502

503

504

505

506

507

508

509 larvae and adults of mayflies: An electrophoretic analysis of three species of Heptageniidae (Ephemeroptera). Italian Journal of Zoology 63:23-30. DOI: 10.1080/11250009609356102.

Semlitsch RD, Anderson TL, Osbourn MS, and Ousterhout BH. 2014. Structure and dynamics of ringed salamander (Ambystoma annulatum) populations in Missouri. Herpetologica 70:14-22. DOI: 10.1655/HERPETOLOGICA-D-13-00074.

Semlitsch RD, Todd BD, Blomquist SM, Calhoun AJK, Gibbons JW, Gibbs JP, Graeter GJ, Harper EB, Hocking DJ, Hunter ML, Patrick DA, Rittenhouse TAG, and Rothermel BB. 2009. Effects of timber harvest on amphibian populations: understanding mechanisms from forest experiments. Bioscience 59:853-862. DOI: 10.1525/bio.2009.59.10.7.

Shoop CR. 1974. Yearly variation in larval survival of Ambystoma maculatum. Ecology 55:440444. DOI: $10.2307 / 1935233$.

Slate J, David P, Dodds KG, Veenvliet BA, Glass BC, Broad TE, and McEwan JC. 2004. Understanding the relationship between the inbreeding coefficient and multilocus heterozygosity: Theoretical expectations and empirical data. Heredity 93:255-265.

Wang J. 2012. Computationally efficient sibship and parentage assignment from multilocus marker data. Genetics 191:183-194. DOI: 10.1534/genetics.111.138149. 
510 Table 1. Summary statistics following COLONY analysis including effective population size

$511\left(\mathrm{~N}_{\mathrm{e}}\right)$ and the proportion of samples removed. The combined life stages sibling removal follows

512 two iterations of COLONY, the first within life stage, the second after pooling life stages.

\begin{tabular}{|c|c|c|c|c|c|c|c|}
\hline \multirow[b]{2}{*}{ Pond } & \multicolumn{3}{|c|}{$\mathrm{N}_{\mathrm{e}}$} & \multicolumn{4}{|c|}{ Proportion of samples removed } \\
\hline & Adult & Embryo & Larvae & Adult & Embryo & Larvae & $\begin{array}{l}\text { Combined } \\
\text { life stages }\end{array}$ \\
\hline 1 & 120 & 92 & 62 & 0.08 & 0.08 & 0.21 & 0.40 \\
\hline 2 & 67 & 114 & - & 0.16 & 0.05 & - & 0.25 \\
\hline 3 & 100 & 93 & - & 0.00 & 0.12 & - & 0.24 \\
\hline 4 & 100 & 55 & 70 & 0.16 & 0.20 & 0.39 & 0.41 \\
\hline 5 & 50 & 58 & 60 & 0.25 & 0.30 & 0.40 & 0.37 \\
\hline Avg & 87.4 & 82.4 & 64 & 0.13 & 0.15 & 0.33 & 0.33 \\
\hline $\mathrm{SD}$ & 25.28 & 22.58 & 4.32 & 0.08 & 0.09 & 0.09 & 0.07 \\
\hline
\end{tabular}


514 Table 2. Rarefied allelic richness and observed heterozygosity estimates at each pond for both the full data set, and with siblings

515 removed. Mixed-tissue is the bootstrap mean and 95\% confidence interval from randomly sampling all life stages together. Bolded 516 type indicates values that fall outside of the bootstrapped $95 \%$ confidence interval.

517 
Full data set

\begin{tabular}{|c|c|c|c|c|c|c|c|c|}
\hline \multirow[b]{2}{*}{ Pond } & \multicolumn{4}{|c|}{$A_{r}$} & \multicolumn{4}{|c|}{$\mathrm{H}_{\mathrm{o}}$} \\
\hline & Adult & Embryo & Larvae & $\begin{array}{l}\text { Mixed samples } \\
(95 \% \mathrm{CI})\end{array}$ & Adult & Embryo & Larvae & $\begin{array}{l}\text { Mixed samples } \\
(95 \% \mathrm{CI})\end{array}$ \\
\hline 1 & 4.11 & 4.22 & 4.21 & $4.22(3.99-4.44)$ & 0.54 & 0.49 & 0.52 & $0.53(0.49-0.57)$ \\
\hline 2 & 3.94 & 4.10 & - & $4.08(3.86-4.27)$ & 0.51 & 0.45 & - & $0.50(0.47-0.53)$ \\
\hline 3 & 3.65 & 3.68 & - & $3.67(3.44-3.89)$ & 0.50 & 0.47 & - & $0.49(0.46-0.53)$ \\
\hline 4 & 3.77 & 3.63 & 3.90 & $3.73(3.50-3.98)$ & 0.52 & 0.50 & 0.49 & $0.51(0.47-0.54)$ \\
\hline 5 & 3.66 & 3.88 & 4.07 & $3.92(3.64-4.17)$ & 0.50 & 0.54 & 0.52 & $0.54(0.50-0.57)$ \\
\hline Avg & 3.83 & 3.90 & 4.06 & $3.93(3.51-4.35)$ & 0.52 & 0.49 & 0.51 & $0.52(0.48-0.56)$ \\
\hline SD & 0.20 & 0.26 & 0.15 & - & 0.01 & 0.03 & 0.02 & - \\
\hline
\end{tabular}

Siblings removed

\begin{tabular}{lllll} 
& & \multicolumn{3}{c}{$\mathrm{A}_{\mathrm{r}}$} \\
\cline { 2 - 5 } Pond & Adult & Embryo & Larvae & $\begin{array}{l}\text { Mixed samples } \\
(95 \% \mathrm{CI})\end{array}$ \\
\hline 1 & 4.16 & 4.20 & 4.36 & $4.22(4.00-4.41)$ \\
2 & 3.98 & 4.15 & - & $4.19(4.05-4.31)$ \\
3 & 3.65 & 3.72 & - & $3.77(3.57-3.95)$ \\
4 & 3.77 & 3.64 & 3.88 & $3.81(3.57-4.03)$ \\
5 & 3.57 & 4.04 & 4.16 & $3.98(3.71-4.21)$ \\
\hline Avg & 3.83 & 3.95 & 4.13 & $3.99(3.62-4.35)$ \\
SD & 0.22 & 0.23 & 0.20 & - \\
\hline
\end{tabular}

\begin{tabular}{|c|c|c|c|}
\hline \multicolumn{4}{|c|}{$\mathrm{H}_{\mathrm{o}}$} \\
\hline Adult & Embryo & Larvae & $\begin{array}{l}\text { Mixed samples } \\
(95 \% \mathrm{CI})\end{array}$ \\
\hline 0.55 & 0.53 & 0.51 & $0.54(0.50-0.57)$ \\
\hline 0.52 & 0.48 & - & $0.51(0.49-0.53)$ \\
\hline 0.50 & 0.48 & - & $0.50(0.47-0.53)$ \\
\hline 0.54 & 0.52 & 0.50 & $0.52(0.49-0.56)$ \\
\hline 0.53 & 0.54 & 0.53 & $0.54(0.51-0.57)$ \\
\hline 0.53 & 0.51 & 0.51 & $0.52(0.48-0.56)$ \\
\hline 0.01 & 0.03 & 0.01 & - \\
\hline
\end{tabular}


519 Table 3. Pairwise genetic distances $\left(F_{\mathrm{ST}}\right.$ and $\left.D_{\mathrm{C}}\right)$ between ponds for each life stage and for mixed-tissue life stages. Mixed is the

520 bootstrap mean and 95\% confidence interval from randomly sampling all life stages together. Bolded type indicates values that fall

521 outside of the bootstrapped $95 \%$ confidence interval.

Full data set

\begin{tabular}{lllll} 
& \multicolumn{4}{c}{$F_{\mathrm{ST}}$} \\
\cline { 2 - 5 } Pond-pair & Adult & Embryo & Larvae & Mixed $(95 \% \mathrm{CI})$ \\
\hline 1_2 & 0.000 & 0.000 & - & $0.003(-0.006-0.014)$ \\
$1 \_3$ & 0.012 & 0.002 & - & $0.011(0.002-0.023)$ \\
$1 \_4$ & 0.019 & 0.020 & 0.020 & $0.008(-0.001-0.020)$ \\
$1 \_5$ & 0.015 & 0.024 & 0.029 & $0.022(0.008-0.037)$ \\
$2 \_3$ & $\mathbf{0 . 0 0 5}$ & $\mathbf{0 . 0 0 6}$ & - & $0.019(0.007-0.032)$ \\
2_4 & 0.011 & $\mathbf{0 . 0 2 3}$ & - & $0.004(-0.006-0.017)$ \\
2_5 & 0.013 & 0.030 & - & $0.022(0.008-0.038)$ \\
$3 \_4$ & $\mathbf{0 . 0 0 0}$ & $\mathbf{0 . 0 0 4}$ & - & $0.019(0.007-0.032)$ \\
$3 \_5$ & 0.012 & $\mathbf{0 . 0 2 3}$ & - & $0.010(0.000-0.022)$ \\
4_5 & 0.005 & 0.012 & 0.011 & $0.010(0.000-0.023)$ \\
\hline Avg & 0.009 & 0.014 & 0.020 & $0.013(0.002-0.026)$ \\
SD & 0.006 & 0.011 & 0.009 & - \\
\hline
\end{tabular}

\begin{tabular}{llll}
\multicolumn{4}{c}{$D_{\mathrm{C}}$} \\
\hline Adult & Embryo & Larvae & Mixed $(95 \% \mathrm{CI})$ \\
\hline 0.168 & 0.195 & - & $0.190(0.163-0.216)$ \\
0.212 & 0.208 & - & $0.235(0.206-0.267)$ \\
0.205 & 0.247 & $\mathbf{0 . 1 9 3}$ & $0.237(0.204-0.268)$ \\
0.227 & 0.250 & $\mathbf{0 . 2 0 4}$ & $0.237(0.208-0.267)$ \\
0.196 & 0.201 & - & $0.209(0.183-0.237)$ \\
$\mathbf{0 . 1 8 8}$ & 0.230 & - & $0.218(0.192-0.244)$ \\
0.216 & 0.233 & - & $0.211(0.183-0.240)$ \\
0.151 & 0.165 & - & $0.171(0.142-0.200)$ \\
0.242 & 0.207 & - & $0.221(0.192-0.250)$ \\
0.213 & 0.197 & $\mathbf{0 . 1 8 1}$ & $0.223(0.194-0.252)$ \\
\hline 0.202 & 0.213 & 0.193 & $0.215(0.187-0.244)$ \\
0.027 & 0.027 & 0.011 & - \\
\hline
\end{tabular}

Siblings removed

\begin{tabular}{lllll} 
& \multicolumn{4}{c}{$F_{\mathrm{ST}}$} \\
\cline { 2 - 5 } Pond-pair & Adult & Embryo & Larvae & Mixed $(95 \% \mathrm{CI})$ \\
\hline 1_2 & 0.000 & 0.000 & - & $0.002(-0.005-0.011)$ \\
1_3 & 0.015 & $\mathbf{0 . 0 0 1}$ & - & $0.011(0.003-0.020)$ \\
1_4 & $\mathbf{0 . 0 2 4}$ & $\mathbf{0 . 0 2 1}$ & $\mathbf{0 . 0 2 4}$ & $0.008(0.000-0.017)$ \\
1_5 & 0.020 & $\mathbf{0 . 0 3 6}$ & 0.028 & $0.017(0.006-0.030)$
\end{tabular}

\begin{tabular}{llll}
\multicolumn{4}{c}{$D_{\mathrm{C}}$} \\
\hline Adult & Embryo & Larvae & Mixed $(95 \% \mathrm{CI})$ \\
\hline 0.191 & 0.199 & - & $0.187(0.164-0.212)$ \\
0.240 & 0.218 & - & $0.230(0.206-0.257)$ \\
0.243 & $\mathbf{0 . 2 5 8}$ & 0.234 & $0.229(0.200-0.256)$ \\
0.242 & $\mathbf{0 . 2 7 3}$ & 0.249 & $0.224(0.195-0.251)$
\end{tabular}




\begin{tabular}{|c|c|c|c|c|c|c|c|c|}
\hline 23 & 0.005 & 0.010 & - & $0.016(0.007-0.027)$ & 0.222 & 0.233 & - & $0.214(0.193-0.234)$ \\
\hline $2 \_4$ & 0.013 & 0.023 & - & $0.006(-0.004-0.018)$ & 0.220 & 0.259 & - & $0.213(0.189-0.235)$ \\
\hline $2 \_5$ & 0.015 & 0.040 & - & $0.019(0.007-0.031)$ & 0.226 & 0.276 & - & $0.203(0.178-0.228)$ \\
\hline 3_4 & 0.000 & 0.006 & - & $0.016(0.007-0.028)$ & 0.179 & 0.191 & - & $0.173(0.146-0.203)$ \\
\hline $3 \_5$ & 0.013 & 0.037 & - & $0.007(-0.001-0.017)$ & 0.262 & 0.256 & - & $0.212(0.189-0.237)$ \\
\hline $4 \_5$ & 0.004 & 0.020 & 0.012 & $0.008(-0.002-0.020)$ & 0.232 & 0.240 & 0.227 & $0.213(0.187-0.239)$ \\
\hline Avg & 0.011 & 0.019 & 0.021 & $0.011(0.002-0.022)$ & 0.226 & 0.240 & 0.237 & $0.210(0.185-0.235)$ \\
\hline SD & 0.008 & 0.014 & 0.007 & - & 0.025 & 0.030 & 0.011 & - \\
\hline
\end{tabular}


523 Table 4. Results of simple Mantel tests assessing the correlation between genetic distance and geographic distance. Mixed life stage

524 represents 1,000 bootstrap iterations, and the corresponding Mantel $r$ and P-value estimates are the mean and 95\% confidence

525 intervals of the bootstrap iterations. Mantel P-values were estimated from 100,000 permutations.

Full data set

\begin{tabular}{llllll} 
& \multicolumn{2}{c}{$F_{\mathrm{ST}}$} & & \multicolumn{2}{c}{$D_{\mathrm{C}}$} \\
\cline { 2 - 3 } \cline { 5 - 6 } Life stage & Mantel $\boldsymbol{r}$ & P-value & & Mantel $\boldsymbol{r}$ & P-value \\
\cline { 1 - 3 } Mixed & $0.38(-0.04-0.71)$ & $0.18(0.03-0.54)$ & & $0.775(0.505-0.943)$ & $0.031(0.016-0.100)$ \\
Adult & 0.715 & 0.034 & & 0.731 & 0.033 \\
Embryo & 0.164 & 0.316 & & 0.687 & 0.033 \\
Larva & -0.125 & 0.666 & & -0.055 & 0.668
\end{tabular}

Siblings removed

\begin{tabular}{|c|c|c|c|c|}
\hline \multirow[b]{2}{*}{ Life stage } & \multicolumn{2}{|c|}{$F_{\mathrm{ST}}$} & \multicolumn{2}{|c|}{$D_{\mathrm{C}}$} \\
\hline & Mantel $r$ & P-value & Mantel $r$ & P-value \\
\hline Mixed & $0.310(-0.10-0.65)$ & $0.22(0.05-0.54)$ & $0.758(0.467-0.936)$ & $0.035(0.016-0.100)$ \\
\hline Adult & 0.704 & 0.033 & 0.794 & 0.016 \\
\hline Embryo & 0.093 & 0.417 & 0.427 & 0.118 \\
\hline Larva & 0.190 & 0.667 & -0.186 & 0.667 \\
\hline
\end{tabular}


527 Figure 1. Map of Daniel Boone Conservation Area depicting the locations of the five ponds used

528 in this study. Wildlife ponds are ponds readily used by amphibians, such as Ambystoma

529 maculatum, for reproduction.

530

531 Figure 2. Bar plots representing mean values of a) observed heterozygosity, b) rarefied allelic

532 richness $\left.\left(\mathrm{A}_{\mathrm{r}}\right), \mathrm{c}\right)$ genetic distance $\left.\left(F_{\mathrm{ST}}\right), \mathrm{d}\right)$ genetic distance $\left(D_{\mathrm{C}}\right)$, e) effective population size

$533\left(\mathrm{~N}_{\mathrm{e}}\right)$, and f) proportion of samples removed due to sibship. Solid bars represent values containing

534 full siblings, patterned bars represent values after sibling removal, and error bars represent

535 standard deviations.

536

537 Figure 3. Change in Mantel $r$ when using $F_{\mathrm{ST}}(\mathrm{a})$ and $D_{\mathrm{C}}(\mathrm{b})$, and the corresponding change in

538 the $\mathrm{P}$-value $\left(\mathrm{c}=F_{\mathrm{ST}} ; \mathrm{d}=D_{\mathrm{C}}\right)$ with increasing proportion of tissue samples coming from larvae

539 and embryos. The dashed line in $\mathrm{c}$ and $\mathrm{d}$ is drawn at 0.05 to indicate the traditional threshold for

540 significance. Mean (solid line) and 95\% confidence intervals (lighter shading) were estimated at

5410.05 increments between from 0 to 1 . A proportion of 0 represents an adult-only sample, while a

542 proportion of 1 represents a larvae/embryo-only sample. At each 0.05 increment, 1000 bootstrap

543 samples were conducted and Mantel P-values were estimated from 100,000 permutations. Each

544 of these statistics was calculated with 5, 10, and 15 microsatellites (full data set), with a different

545 set of microsatellites being randomly selected at each bootstrap iteration.

546

547 Figure 3. Observed heterozygosity and allelic richness, averaged over all populations. Mean 548 (solid line) and 95\% confidence intervals (lighter shading) were estimated at 0.05 increments

549 between from 0 to 1 . A proportion of 0 represents an adult-only sample, while a proportion of 1

550 represents a larvae/embryo-only sample. At each 0.05 increment, 1000 bootstrap samples were 
551 conducted. Each of these statistics was calculated with 5, 10, and 15 microsatellites (full data

552 set), with a different set of microsatellites being randomly selected at each bootstrap iteration. 553 


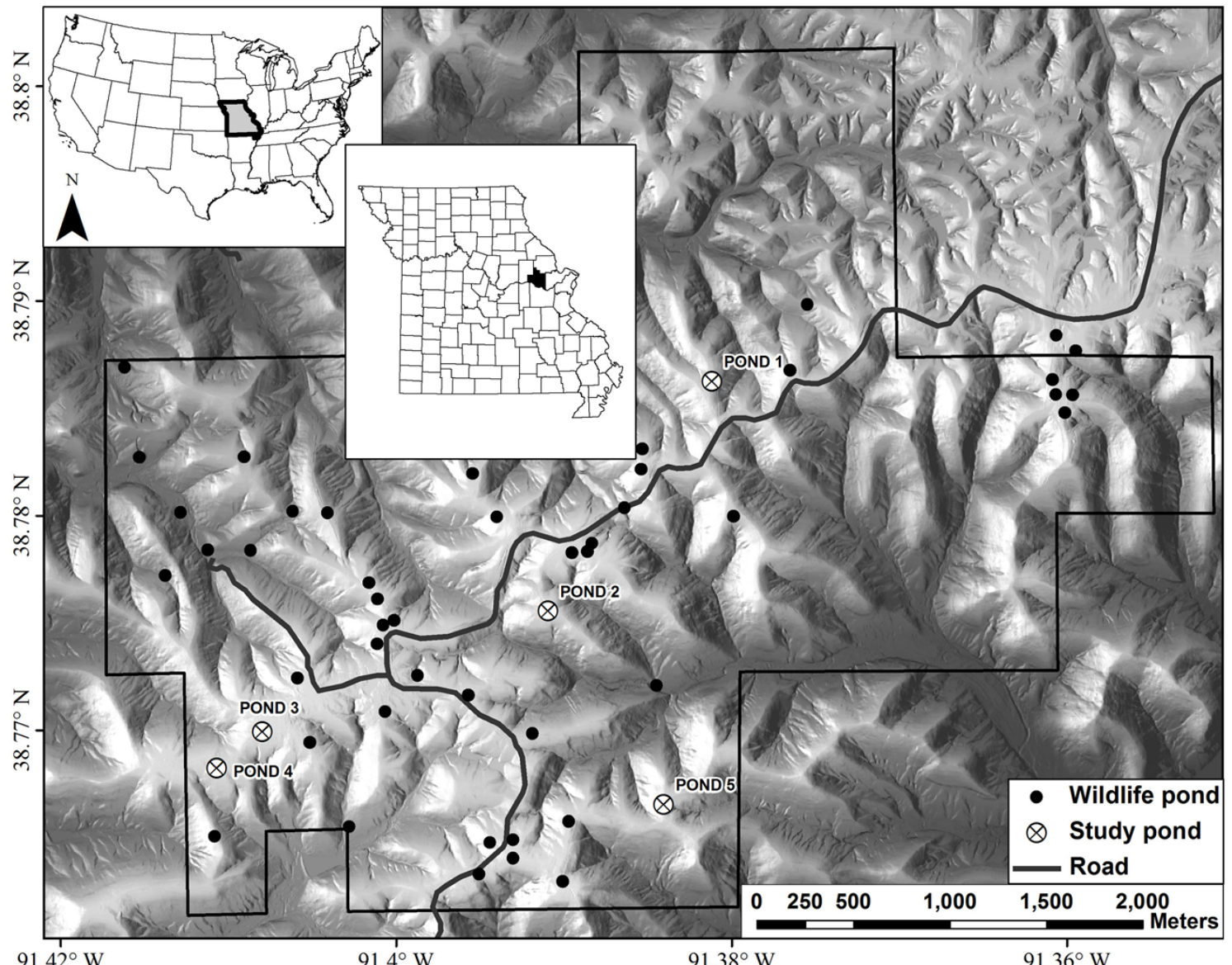

555 Fig. 1 

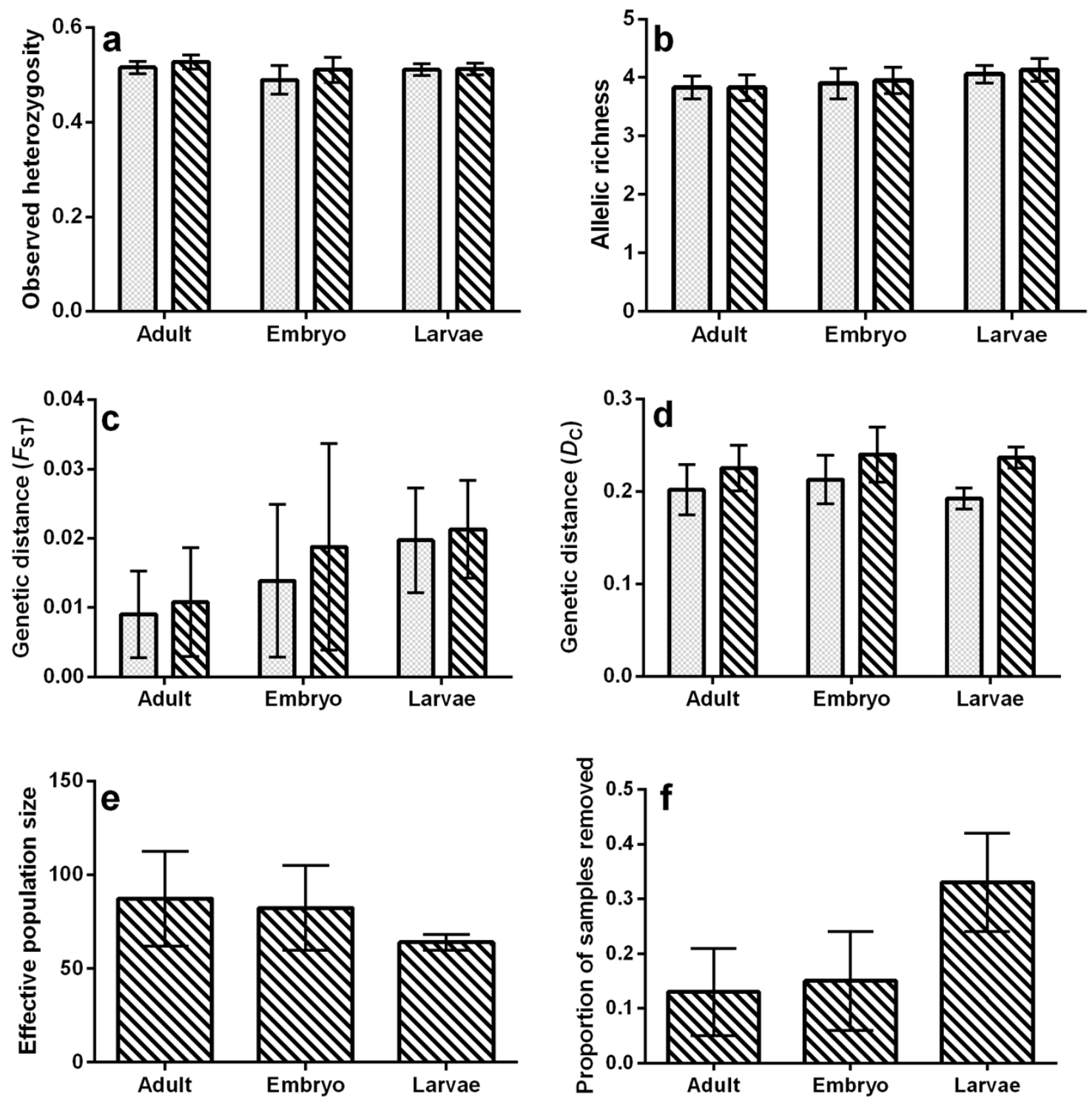

557 Fig. 2 

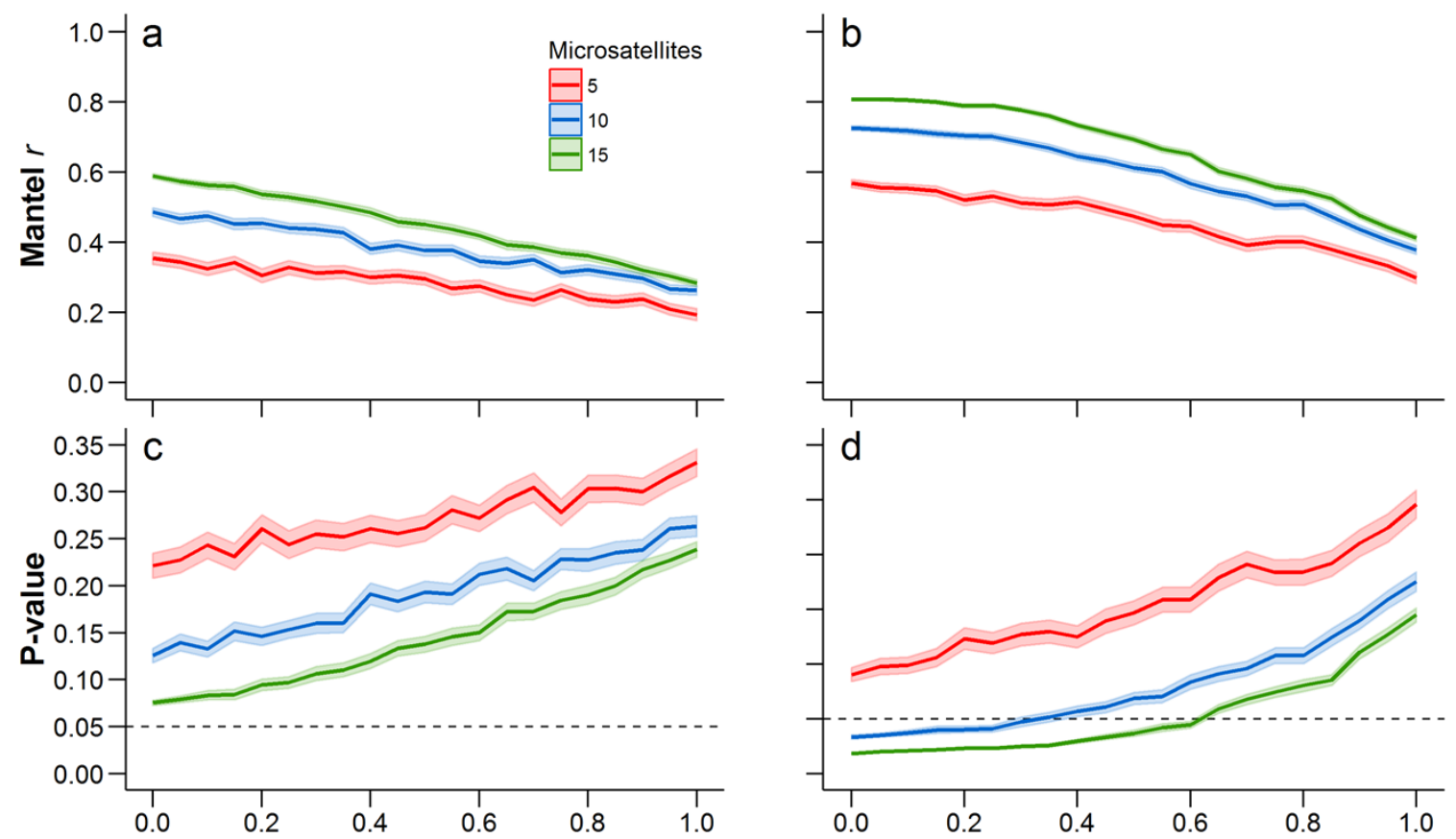

558

Proportion of samples from larvae/embryos

559 Fig. 3 

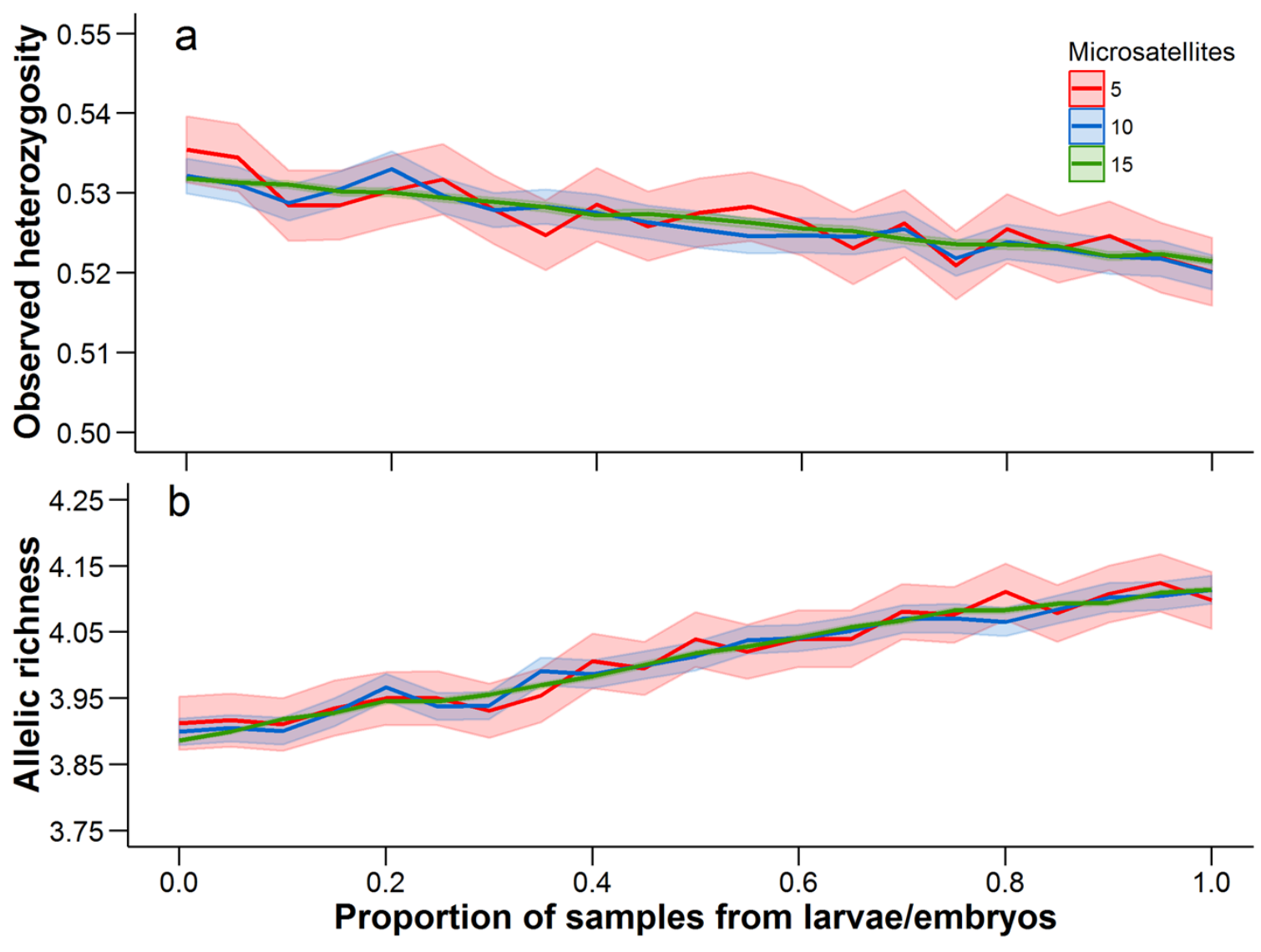

560

561 Fig. 4 